\title{
Analysis of thin-walled beams with variable monosymmetric cross section by means of Legendre polynomials
}

https://doi.org/10.2478/sgem-2019-0001

received November 20, 2018; accepted January 2, 2019.

Abstract: This article deals with the vibrations of a nonprismatic thin-walled beam with an open cross section and any geometrical parameters. The thin-walled beam model presented in this article was described using the membrane shell theory, whilst the equations were derived based on the Vlasov theory assumptions. The model is a generalisation of the model presented by Wilde (1968) in 'The torsion of thin-walled bars with variable cross-section', Archives of Mechanics, 4, 20, pp. 431-443. The Hamilton principle was used to derive equations describing the vibrations of the beam. The equations were derived relative to an arbitrary rectilinear reference axis, taking into account the curving of the beam axis and the axis formed by the shear centres of the beam cross sections. In most works known to the present authors, the equations describing the nonprismatic thin-walled beam vibration problem do not take into account the effects stemming from the curving (the inclination of the walls of the thin-walledcross section towards to the beam axis) of the analysed systems. The recurrence algorithm described in Lewanowicz's work (1976) 'Construction of a recurrence relation of the lowest order for coefficients of the Gegenbauer series', Applicationes Mathematicae, XV(3), pp. 345-396, was used to solve the derived equations with variable coefficients. The obtained solutions of the equations have the form of series relative to Legendre polynomials. A numerical example dealing with the free vibrations of the beam was solved to verify the model and the effectiveness of the presented solution method. The results were compared with the results yielded by finite elements method (FEM).

\footnotetext{
*Corresponding author: Józef Szybiński, Wrocław University of Science and Technology, Faculty of Civil Engineering, Wroctaw, Poland, E-mail: jozef.szybinski@pwr.edu.pl

Piotr Ruta: Wroctaw University of Science and Technology, Faculty of Civil Engineering, Wroctaw, Poland
}

Keywords: thin-walled; free vibrations; nonprismatic; Legendre polynomials.

\section{Introduction}

The problem of the free vibrations of nonprismatic thinwalled beam systems is interesting for two reasons. The first reason is the need to describe more precisely and solve this mathematically difficult problem that, except for special cases, has no closed analytical solutions. The second reason is practical and stems from the necessity to rationally shape and economically design contemporary civil engineering structures built from thin-walled beams with variable geometrical and material parameters.

The problems relating to thin-walled beam systems have been studied by many authors. As the literature on this subject is very extensive, only the research works that have been published in the recent years are presented here. Eisenberger (1997). Torsional vibrations of open and variable cross-section bars. Thin-Walled Structures. 28(3-4), 269-278. analysed the torsional free vibrations of a nonprismatic I-beam. Using the classical power series to approximate the sought functions, he determined the free vibration frequencies with and without the warping effect taken into account. On the basis of Vlasov's theory of thinwalled systems, Ambrosini et al. (2000). A modified Vlasov theory for dynamic analysis of thin-walled and variable open section beams. Engineering Structures. 22(8), 890-900. DOI: 0.1016/S0141-0296(99)00043-7. presented equations describing the vibrations of a nonprismatic thin-walled beam with an open cross section, which take into account the effects of shear flexibility and rotatory inertia in the stress resultants. The same beam model was used by F. de Borbón and Ambrosini (2010). On free vibration analysis of thin-walled beams axially loaded. Thin-Walled Structures. 48(12), 915-920. DOI: 10.1016/j.tws.2010.06.002. to solve the free vibration problem with the influence of the static axial forces taken into account. The derived equations were solved using the method referred to as the state variables 
approach. The eigenproblem was also analysed by Arpaci and Bozdag (2002). On free vibration analysis of thinwalled beams with nonsymmetrical open cross-sections. Computers and Structures. 80(7-8), 691-695. The subject of the considerations was the vibrations of a prismatic beam with any cross section. The problem was solved analytically. The problem of the free vibrations of I-girders with intermediate bracings was analysed by Nguyen et al. (2011). Natural frequency for torsional vibration of simply supported steel I-girders with intermediate bracings. Thin-Walled Structures. 49(4), 534-542. DOI: 10.1016/j. tws.2010.12.001. Using trigonometric functions for approximation, they obtained a closed solution. Heyligerin (2015). Elasticity-based free vibration of anisotropic thinwalled beams. Thin-Walled Structures. 95:73-87. DOI: 10.1016/j.tws.2015.06.014. analysed the eigenproblem of prismatic thin-walled beams with, respectively, an open and a closed cross sections, taking into account anisotropy features. The derived equations were solved using the Ritz method, whilst polynomials and trigonometric functions were used to approximate the sought functions. Dynamic equations of the equilibrium of nonprismatic thinwalled beams with, respectively, an open and a closed cross sections were derived using Hamilton's principle by Chang-New Chen (1998). Variational derivation of the dynamic equilibrium equations of nonprismatic thinwalled beams defined on an arbitrary coordinate system. Mechanics of Structures and Machines. 26(2),219-237. The equations were derived relative to an arbitrarily adopted axis, assuming the rotation of the cross sections relative to this axis. The obtained equilibrium equations and the boundary conditions are strongly conjugated. The problem of the warping of I-bars nonsymmetric in their cross section was solved by Mohri et al. in Mohri, F., Brouki, A. \& Roth, J. C. (2003). Theoretical and numerical stability analyses of unrestrained, mono-symmetric thin-walled beams. Journal of Constructional Steel Research. 59(1), 63-90., where the effect of the load application point on the critical load value was investigated. The derived equations were solved using the Ritz method and the Galerkin method and trigonometric functions for approximation. The warping problem was also considered by Asgarian et al. (2013). Lateral-torsional buckling of tapered thin-walled beams with arbitrary cross-sections. Thin-Walled Structures. 62,96108. DOI: 10.1016/j. tws.2012.06.007. who used Hamilton's principle to derive general equations of equilibrium describing a nonprismatic beam under the assumption that the beam cross section rotates relative to the centre of shear. The obtained differential equations were solved using the classical power series for approximation. Warping and free vibrations were also the subject of work [20] by Soltani et al. The same authors (2014b). Finite element method for stability and free vibration analyses of non-prismatic thinwalled beams. Thin-Walled Structures. 82, 245- 261. DOI: 10.1016/j.tws.2014.04.012. formulated a nonprismatic thinwalled finite element. In order to determine the exact shape functions, they used the method described previously in Asgarian, B., Soltani, M. \& Mohri, F. (2013). Lateraltorsional buckling of tapered thin-walled beams with arbitrary cross-sections. Thin-Walled Structures. 62,96108. and [20]. The formulated finite elements were used to analyse the eigenproblem and the stability problem for nonprismatic beams with any cross section. Similar to the previous works, the results were compared with the ones obtained using finite elements method (FEM). The works by Machado and Cortínez Mohri (2005). Non-linear model for stability of thin-walled composite beams with shear deformation. Thin-Walled Structures. 43(10),1615-1645. DOI: 10.1016/j.tws.2005.06.008. and Mohri et al. Mohri, F., Damil, N. \& Ferry, M. P. (2008). Large torsion finite element model for thin-walled beams. Computers and Structures. 86(7-8), 671-683. DOI: 10.1016/j.compstruc.2007.07.007. are examples of the application of nonlinear analysis to thin-walled beam stability problems. In Machado, S. P. \& Cortínez, V. H. (2005). Non-linear model for stability of thin-walled composite beams with shear deformation. Thin-Walled Structures. 43(10),1615-1645., the stability problem, including the behaviour after stability loss (buckling), was analysed for composite thin-walled beams with, respectively, an open and a closed cross sections. The nonlinear system of equations was solved by means of the incremental Newton-Raphson method. In Mohri, F., Damil, N. \& Ferry, M. P. (2008). Large torsion finite element model for thin-walled beams. Computers and Structures. 86(7-8), 671-683., a nonlinear finite element with two joints and seven degrees of freedom was formulated and used to analyse the post-buckling behaviour of a thinwalled beam. The nonlinear equations stemming from this problem were solved using the iterative-incremental Newton-Raphson method. Extensive literature on the vibrations and stability of thin-walled beams can be found in, amongst others, the already cited work Asgarian, B., Soltani, M. \& Mohri, F. (2013). Lateral-torsional buckling of tapered thin-walled beams with arbitrary cross-sections. Thin-Walled Structures. 62,96-108..

The subject of this article is the problem of the free vibrations of a nonprismatic monosymmetric beam with any parameters described using the membrane shell theory and the Vlasov theory assumptions. The beam model presented here is a generalisation of the model found in Wilde's article [22]. The generalisation consisted in taken into account the curving of the axis formed by the shear 
centres of the beam cross sections. In most works on the vibrations of thin-walled beams known to the present authors, the equations describing this problem do not take into account the effects stemming from the curvilinear form of the beam axis and the axis defined by the shear centres. The Hamilton principle was used to derive equations describing the vibrations of the beam. In this article, the equations describing the vibrations of the beam were derived relative to an arbitrary rectilinear reference axis and the effects stemming from the curvilinear form of the axes were taken into account in the equations.

An approximation method using Legendre's orthogonal polynomials (a special type of the Gegenbauer polynomials) for approximation was used to solve the obtained differential equations. This method, presented by Lewanowicz in, amongst others, work [9], so far has not been applied to this kind of problems. It is a generalisation of the method of approximating the solution of the Chebyshev series of the first kind [see Paszkowski Paszkowski, S. (1975). Numerical applications of Chebyshev polynomials. Warsaw: PWN; (in Polish).) and used by the present authors in earlier articles Ruta, P. \& Szybiński, J. (2014). Nonlinear analysis of nonprismatic Timoshenko beam for different geometric nonlinearity models. International Journal of Mechanical Sciences. 101102, 349-362. DOI: 10.1016/j. ijmecsci.2015.07.020. In the case of the free vibration problem, this method reduces the system of ordinary differential equations to an infinite system of algebraic equations.

A calculation example is provided to verify the derived equations and illustrate the capabilities of the presented method. In the example, the eigenproblem of a beam with a linearly variable web height is solved. Two static schemes were considered: (1) a cantilever beam and (2) a clamped-clamped beam. The effect of omitting certain parameters connected with the variability of the beam geometry was examined. The obtained eigenfrequencies were compared with the results yielded by FEM (Abaqus and SOFiSTiK).

\section{Problem formulation}

The subject of the considerations is the problem of the spatial free vibrations of an arbitrarily supported, nonprismatic, thin-walled beam.

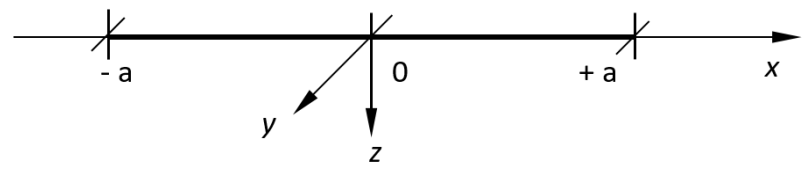

Figure 1: Static scheme of considered system.

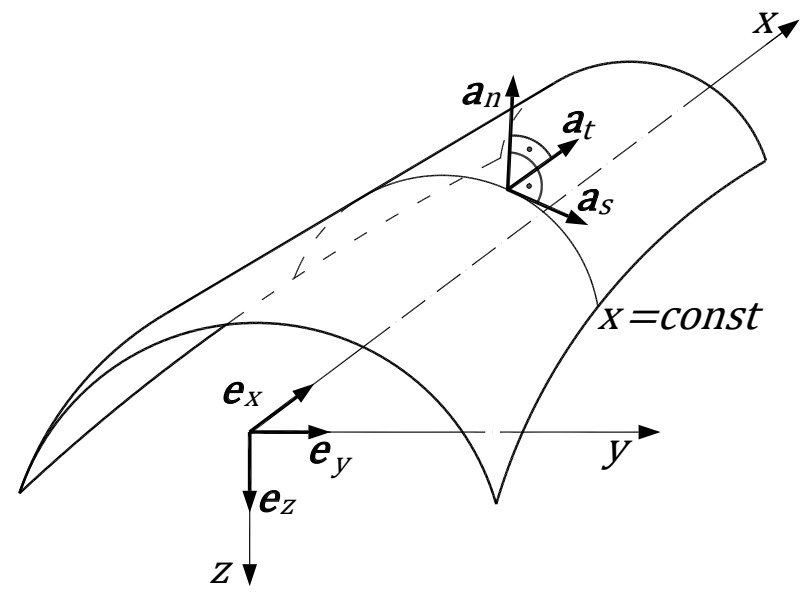

Figure 2: Coordinate system and local basis vectors for nonprismatic thin-walled beam.

\subsection{Description of model}

The description of the model presented in this article is based on the membrane shell theory and the Vlasov assumptions used in the theory of thin-walled systems. The model is a generalisation of the model described by Wilde in [22]. The generalisation consists in taken into account the fact that the coordinates defining the position of the shear centres form a curvilinear axis and depend on the coordinate describing the position of the analyzed cross section. Also the beam axis is curvilinear. As a nomenclature different from that of Wilde's work [22] is used in this article, the excerpts from the original work quoted in this section have been modified using the new nomenclature.

The displacement equations used here were derived relative to a rectilinear reference axis and to the system of coordinates $x, y, z$ (Fig. 2). At this stage, no additional simplifying assumptions concerning the beam's geometry (e.g. symmetry) were adopted.

The vector describing the location of a point on the central surface of the shell is expressed using the formula

$$
\boldsymbol{r}=t \boldsymbol{e}_{x}+y(t, s) \boldsymbol{e}_{y}+z(t, s) \boldsymbol{e}_{z}
$$


where $e_{x}, e_{y}, e_{z}$, are the versors of the cartesian coordinate system basis.

The vectors of the covariant basis of the system of coordinates $(t, s, n)$, determined on the central surface of the shell are defined as follows:

$$
\boldsymbol{a}_{t}=\boldsymbol{e}_{x}+y_{, t} \boldsymbol{e}_{y}+z_{, t} \boldsymbol{e}_{z}, \boldsymbol{a}_{s}=y_{, s} \boldsymbol{e}_{y}+z_{, s} \boldsymbol{e}_{z,}
$$

where $f_{, s}=\partial f(t, s) / \partial s, f_{, t}=\partial f(t, s) / \partial t$.

Coordinate describes the arc length and so $\left(y_{s}\right)^{2}+\left(z_{s}\right)^{2}=1$.

Therefore, one can introduce the following denotations $\mathrm{y}_{\mathrm{s}}=\cos \alpha, \mathrm{z}_{\mathrm{s}}=\sin \alpha$, where $\alpha$ is the angle included between the positive part of axis $y$ and the tangent to the cross section when $t=$ const. The third unit vector of the local basis defines the following relation:

$$
\boldsymbol{a}_{n}=\frac{-\boldsymbol{a}_{t} \times \boldsymbol{a}_{5}}{\left|\boldsymbol{a}_{t} \times \boldsymbol{a}_{s}\right|}=\left(1+\beta^{2}\right)^{-\frac{1}{2}}\left(\beta \boldsymbol{e}_{x}+\sin \alpha \boldsymbol{e}_{y}-\cos \alpha \boldsymbol{e}_{z}\right)
$$

where $\beta=-y_{t} \sin \alpha+z_{t} \cos \alpha$. Thus the matrix for the transformation of the Cartesian coordinate system basis to the covariant local basis of the system defined on the central surface is given by the formula

$$
\boldsymbol{C}_{i .}^{k}=\left[\begin{array}{ccc}
1 & y_{, t} & z_{, t} \\
0 & \cos \alpha & \sin \alpha \\
\frac{\beta}{\sqrt{1+\beta^{2}}} & \frac{\sin \alpha}{\sqrt{1+\beta^{2}}} & \frac{-\cos \alpha}{\sqrt{1+\beta^{2}}}
\end{array}\right]
$$

For the local coordinate system tied to the central surface, the covariant surface metric tensor is expressed using the following formula:

$$
\begin{gathered}
\boldsymbol{a}_{\alpha \beta}=\left[\begin{array}{cc}
1+\beta^{2}+\gamma^{2} & \gamma \\
\gamma & 1
\end{array}\right], \\
\left|\boldsymbol{a}_{\alpha \beta}\right|=1+\beta^{2},
\end{gathered}
$$

where $\gamma=y_{, t} \cos \alpha+z_{, t} \sin \alpha$.

The covariant metric tensor assumes the following form:

$$
\boldsymbol{a}^{\alpha \beta}=\left[\begin{array}{cc}
\frac{1}{1+\beta^{2}} & -\frac{\gamma}{1+\beta^{2}} \\
-\frac{\gamma}{1+\beta^{2}} & \frac{1+\beta^{2}+\gamma^{2}}{1+\beta^{2}}
\end{array}\right]
$$

In general, the defined surface local coordinate system is not orthogonal. On the basis of the system defined earlier, one can define an orthogonal coordinate system as follows:

$$
\begin{aligned}
& \boldsymbol{a}_{S}=\boldsymbol{a}_{S}, \boldsymbol{a}_{N}=\boldsymbol{a}_{n} \\
& \boldsymbol{a}_{S} \perp \boldsymbol{a}_{T} \perp \boldsymbol{a}_{n},\left|\boldsymbol{a}_{T}\right|=1
\end{aligned}
$$

From the above relations, it follows that versor $\boldsymbol{a}_{T}$ lies in the plane tangent to the surface. Therefore, the vector

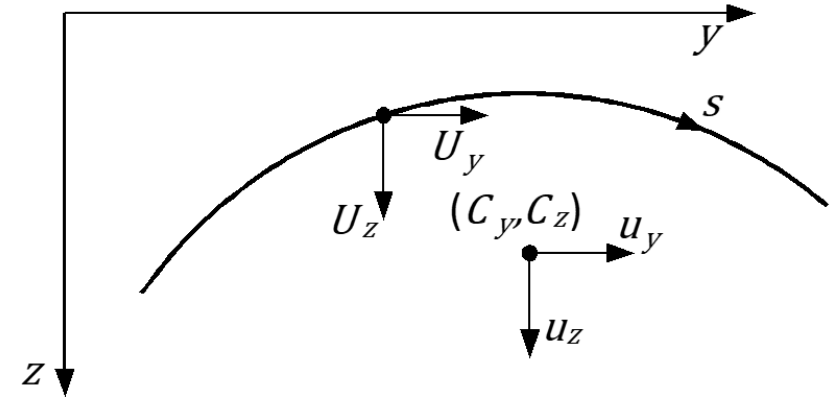

Figure 3: Description of displacements for cross section.

is defined as $\boldsymbol{a}_{T}=\alpha_{1} \boldsymbol{a}_{t}+\alpha_{2} \boldsymbol{a}_{s}$. The values of coefficients $\alpha_{1}$ and $\alpha_{2}$ were calculated from the relation $\boldsymbol{a}_{T} \perp \boldsymbol{a}_{S},\left|\boldsymbol{a}_{T}\right|$ $=1$. Ultimately, the matrix for the transformation of local coordinate system $\boldsymbol{a}_{s}, \boldsymbol{a}_{t}$ to a system of orthogonal vectors $\boldsymbol{a}_{S}, \boldsymbol{a}_{T}$ lying on the surface tangent to the central surface of the shell is defined using the formula

$$
\boldsymbol{C}_{\Omega}^{\alpha}=\left[\begin{array}{cc}
\frac{1}{\sqrt{1+\beta^{2}}} & -\frac{\gamma}{\sqrt{1+\beta^{2}}} \\
0 & 1
\end{array}\right]
$$

and the inverse matrix has the form

$$
\overline{\boldsymbol{C}}_{\alpha .}^{\Omega}=\left[\begin{array}{cc}
\sqrt{1+\beta^{2}} & \gamma \\
0 & 1
\end{array}\right] .
$$

Let us assume that the centre lines of the beam cross sections undergo deformation only in the direction consistent with the direction of the longitudinal axis $x$ (the so-called rigid cross section hypothesis). This leads to the following equation describing the displacement of an arbitrary point of the thin-walled beam:

$$
\begin{gathered}
\boldsymbol{u}=U_{x} \boldsymbol{e}_{x}+U_{y} \boldsymbol{e}_{y}+U_{z} \boldsymbol{e}_{z}= \\
=u_{x}(t, s) \boldsymbol{e}_{x}+\left[u_{y}(t)-\left(z(t, s)-C_{z}(t)\right) \theta(t)\right] \boldsymbol{e}_{y} \\
+\left[u_{z}(t)+\left(y(t, s)-C_{y}(t)\right) \theta(t)\right] \boldsymbol{e}_{z}
\end{gathered}
$$

where $\theta(t)$ is the angle of rotation relative to the axis marked out by the points whose coordinates $\left(C_{y}(\mathrm{t}), C_{z}(\mathrm{t})\right)$ define the centre of shear, $u_{y}(t), u_{z}(t)$ are the components of the displacements of the cross section in the shear centre measured along the axis of the global coordinate system and $u_{x}(t, s)$ is the measure of the displacements of the cross section along axis $x$, taking into account wrappings relative to plane $x=$ const.

The linear components of the deformation tensor are expressed using the formula

$$
\gamma_{\alpha \beta}=\frac{1}{2}\left(\boldsymbol{a}_{\alpha} \cdot \boldsymbol{u}_{, \beta}+\boldsymbol{a}_{\beta} \cdot \boldsymbol{u}_{, \alpha}\right) .
$$


Hence,

$$
\begin{gathered}
\gamma_{t t}=u_{x, t}+y_{, t}\left(u_{y, t}-\left(z-C_{z}\right) \theta_{, t}+C_{z, t} \theta\right)+z_{, t}\left(u_{z, t}+\left(y-C_{y}\right) \theta_{t, t}-C_{y, t} \theta\right) \\
\gamma_{t s}=\gamma_{s t}=\frac{1}{2}\left(u_{x, s}+y_{s}\left(u_{y, t}-\left(z-C_{z}\right) \theta_{, t}+C_{z, t} \theta\right)+z_{, s}\left(u_{z, t}+\left(y-C_{y}\right) \theta_{t, t}-C_{y, t} \theta\right)\right) \\
\gamma_{s s}=0
\end{gathered}
$$

The physical deformations are expressed using the formula

$$
\gamma_{\Omega \Phi}=C_{\Omega}^{\alpha}, C_{\Phi,}^{\beta} \gamma_{\alpha \beta}
$$

Using formula (9), one gets

$$
\begin{gathered}
\gamma_{T T}=\left(1+\beta^{2}\right)^{-1} \gamma_{t t}-2 \gamma\left(1+\beta^{2}\right)^{-1} \gamma_{t s} \\
\gamma_{T S}=\gamma_{S T}=\left(1+\beta^{2}\right)^{-\frac{1}{2}} \gamma_{t s}, \gamma_{S S}=0 .
\end{gathered}
$$

According to the Vlasov assumption, strain $\gamma_{T S}=0$. Thus it follows from formula (14) that also strain $\gamma_{t s}=0$. This assumption ultimately leads to the following relation:

$$
u_{x, s}=-\left(y_{, s}\left(u_{y, t}-\left(z-C_{z}\right) \theta_{, t}+C_{z, t} \theta\right)+z_{s}\left(u_{z, t}+\left(y-C_{y}\right) \theta_{, t}-C_{y, t} \theta\right)\right)
$$

Integrating both sides of equation (15) over variable $s$ and taking into account the fact that on the right side of this equality, only functions $z$ and $y$ depend on $s$, one gets

$$
u_{x}=u_{0 x}-y u_{y, t}-z u_{z, t}-\left(y C_{z, t}-z C_{y, t}\right) \theta-\omega \theta_{, t}
$$

where $u_{0 x}=u_{0 x}(t)$ is an integration constant independent of variable $s$ and $\omega=\omega(t, s)$ is a sectorial coordinate described by the following relation:

$$
\omega(t, s)=\int\left[-\left(z-C_{z}\right) y_{, s}+\left(y-C_{y}\right) z_{,}\right] d s
$$

Then performing the differentiation of function $u_{x}(16)$ over variable $t$, one gets

$$
\begin{gathered}
u_{x, t}=u_{0 x, t}-y_{, t} u_{y, t}-y u_{y, t t}-z_{t, t} u_{z, t}-z u_{z, t t}-\left(y_{t} C_{z, t}+y C_{z, t t}-z_{, t} C_{y, t}-z C_{y, t t}\right) \theta- \\
\left(y C_{z, t}-z C_{y, t}\right) \theta_{, t}-\omega_{, t} \theta_{, t}-\omega \theta_{, t t}
\end{gathered}
$$

Substituting $u_{x, t}$ into formula (12) defining $\gamma_{t t}$, and taking into account the fact that $t=x$, ultimately, one gets

$$
\gamma_{x x}=u_{0 x, x}-y u_{y, x x}-z u_{z, x x}-\omega \theta_{, x x}-\psi \theta_{, x}-\delta \theta,
$$

where

$$
\begin{gathered}
\psi=\omega_{, x}+y_{, x}\left(z-C_{z}\right)-z_{, x}\left(y-C_{y}\right)+\left(y C_{z, x}-z C_{y, x}\right), \\
\delta=y C_{z, x x}-z C_{y, x x} .
\end{gathered}
$$

The dependences between strains and stresses are defined using the formula

$$
n^{\alpha \beta}=D \boldsymbol{H}^{\alpha \beta \varrho \lambda} \gamma_{\varrho \lambda}
$$

where $\quad \boldsymbol{H}^{\alpha \beta \varrho \lambda}=\frac{1}{2}\left[\boldsymbol{a}^{\alpha \beta} \boldsymbol{a}^{\beta \varrho}+\boldsymbol{a}^{\alpha \varrho} \boldsymbol{a}^{\beta \lambda}+v\left(\boldsymbol{\varepsilon}^{\alpha \varrho} \boldsymbol{\varepsilon}^{\beta \lambda}+\boldsymbol{\varepsilon}^{\alpha \lambda} \boldsymbol{\varepsilon}^{\beta \varrho}\right)\right]$, $D=E^{\prime} g, E^{\prime}=\frac{E}{1-v^{2}}, v$ is the Poisson ratio, $E$ is Young's modulus, $g$ is the wall thickness and $\boldsymbol{\varepsilon}^{\alpha \beta}$ is the Ricci tensor. In the considered case, relation (21), after taking into account the fact that $\gamma_{x s}=\gamma_{s s}=0$ is, comes down to

$$
n^{x x}=E^{\prime} g\left(1+\beta^{2}\right)^{-2} \gamma_{x x} .
$$

Let us consider cross-sectional forces acting in cross section $x=$ const. The force vector defining the membrane forces was determined from the relation

$$
\boldsymbol{t}=n^{\alpha \beta} n_{\alpha} \boldsymbol{a}_{\beta}
$$

where $n_{a}$ is a covariant element of the unit vector normal to the plane and perpendicular to the line tangent to the cross section. Thus $\boldsymbol{n}$ is equal to $\boldsymbol{a}_{T}$, and so the following equalities hold: $n_{x}=\left(1+\beta^{2}\right)^{\frac{1}{2}}, n_{s}=0$. Hence,

$$
\boldsymbol{t}=n^{x x}\left(1+\beta^{2}\right)^{\frac{1}{2}} \boldsymbol{a}_{t}+n^{x s}\left(1+\beta^{2}\right)^{\frac{1}{2}} \boldsymbol{a}_{s}
$$

In order to find component $\boldsymbol{t}$ acting along axis $x$, one projects vector $\boldsymbol{t}$ onto axis $x$ by scalar multiplying it by vector $\boldsymbol{e}_{x}$. Ultimately, one gets

$\boldsymbol{t}_{x}=\boldsymbol{t} \cdot \boldsymbol{e}_{x}=E^{\prime} g^{*}\left[u_{0 x, \mathrm{x}}-y u_{y, \mathrm{xx}}-z u_{z, \mathrm{xx}}-\omega \theta_{, \mathrm{xx}}-\psi \theta_{, x}-\delta \theta\right]$

where $g^{*}=\left(1+\beta^{2}\right)^{-\frac{3}{2}} g$ expresses the so-called reduced thickness.

The internal forces are defined using the formulas

$N=\int_{S} \boldsymbol{t}_{\boldsymbol{x}} d s, \quad M_{y}=\int_{S} z \boldsymbol{t}_{\boldsymbol{x}} d s, \quad M_{z}=\int_{S} y \boldsymbol{t}_{\boldsymbol{x}} d s, \quad B=\int_{S} \omega \boldsymbol{t}_{\boldsymbol{x}} d s$

where integration is performed over the contour of the cross section. Substituting relation (25) into formula (26) and performing the integrations, one gets the following formulas for the cross-sectional forces (under the assumption that the considered beam is monosymmetric and that axis $z$ is the symmetry axis of the cross sections): 


$$
\begin{gathered}
N=E^{\prime}\left[A^{*} u_{0 x, x}-S_{y}^{*} u_{z, x x}\right] \\
M_{y}=E^{\prime}\left[S_{y}^{*} u_{0 x, x}-I_{y}^{*} u_{z, x x}\right] \\
M_{z}=E^{\prime}\left[-I_{z}^{*} u_{y, x x}-I_{\psi z}^{*} \theta_{, x}-I_{\delta z}^{*} \theta\right] \\
B=E^{\prime}\left[-I_{\omega}^{*} \theta_{, x x}-I_{\psi \omega}^{*} \theta_{, x}-I_{\delta \omega}^{*} \theta\right]
\end{gathered}
$$

where

$$
\begin{gathered}
A^{*}=\int_{S} d A^{*}, S_{y}^{*}=\int_{S} z d A^{*}, \\
I_{y}^{*}=\int_{S} z^{2} d A^{*}, I_{z}^{*}=\int_{S} y^{2} d A^{*}, \\
I_{\omega}^{*}=\int_{S} \omega^{2} d A^{*}, \\
I_{\psi z}^{*}=\int_{S} y \psi d A^{*}, I_{\psi \omega}^{*}=\int_{S} \omega \psi d A^{*}, \\
I_{\delta z}^{*}=\int_{S} y \delta d A^{*}, I_{\delta \omega}^{*}=\int_{S} \omega \delta d A^{*} .
\end{gathered}
$$

In order to simplify the notation in formula (28), denotation $d A^{*}=g^{*} d s$, in which $g^{\star}$ is reduced thickness $g^{*}=\left(1+\beta^{2}\right)^{-\frac{3}{2}} g$, was used.

\subsection{Displacement equations}

In order to derive displacement equations describing the vibration problem for a thin-walled beam, we shall use the Hamilton principle

$$
\delta\left(U_{S}-U_{M}\right)=\delta U_{S}-\delta U_{M}=0
$$

where $U_{S}$ is the potential energy of the elastic deformation (the linear part) and $U_{M}$ is the kinetic energy of the system. The potential energy of the elastic deformation is expressed using the formula

$$
U_{S}=\frac{1}{2} \iint n^{\alpha \beta} \gamma_{\alpha \beta} d S+\frac{1}{2} \int_{-a}^{a} G I_{S}\left(\theta_{, x}\right)^{2} d x,
$$

where $G I_{s}$ is the stiffness in the Saint Venant torsion. Considering the relations

$$
\begin{gathered}
\frac{1}{2} \iint n^{\alpha \beta} \gamma_{\alpha \beta} d S=\frac{1}{2} \iint n^{t t} \gamma_{t t} d S, \\
n^{x x}=E^{\prime} g\left(1+\beta^{2}\right)^{-2} \gamma_{x x}, \\
d S=\sqrt{a} d s d x=\sqrt{1+\beta^{2}} d s d x,
\end{gathered}
$$

the potential energy of the system can be presented in the form

$$
U_{S}=\frac{1}{2} \iint E^{\prime} \gamma_{x x}^{2} d A^{*} d x+\frac{1}{2} \int_{-a}^{a} G I_{S}\left(\theta_{, x}\right)^{2} d x
$$

where $A^{*}=g^{*} d s, g^{*}=\left(1+\beta^{2}\right)^{-\frac{3}{2}} g$. Taking relation (12) into account in formula (32), one gets

$$
\begin{aligned}
& U_{S}=\int_{-a}^{a}\left\{\frac{1}{2} E^{\prime} A^{*} u_{0 x, x}^{2}+l_{z}^{*} u_{y, x x}^{2}+l_{y}^{*} u_{z, x x}^{2}+l_{\omega}^{*} \theta_{x, x}^{2}+l_{\psi}^{*} \theta_{x x}^{2}+l_{\delta}^{*} \theta^{2}-2 S_{y}^{*} u_{0 x x} u_{z, x x}+2 l_{\psi z}^{*} u_{y x x} \theta_{, x}\right. \\
& \left.\left.+2 \int_{\delta z z}^{*} u_{y, x} \theta+2 \Gamma_{\psi \omega}^{*} \theta_{x x} \theta_{x}+2 I_{\delta \omega}^{*} \theta_{x x} \theta+2 I_{\delta \psi}^{*} \theta_{x} \theta\right]+\frac{1}{2} G l_{s}\left(\theta_{x}\right)^{2}\right\} d x
\end{aligned}
$$

The kinetic energy is expressed using the formula

$$
U_{M}=\frac{1}{2} \iint \rho\left(\dot{U}_{x}^{2}+\dot{U}_{y}^{2}+\dot{U}_{z}^{2}\right) g d S,
$$

where $\dot{U}_{x}^{2}, \dot{U}_{y}^{2}, \dot{U}_{z}^{2}$ are velocities of an arbitrary point of the cross section and $g d S=g \sqrt{a} d s d x=g \sqrt{1+\beta^{2}} d s d x$. After substituting relation (10) into formula (34) and performing transformations, one gets the following final form of the equation for the kinetic energy of the system:

$$
\begin{gathered}
U_{M}=\frac{1}{2} \int_{-a}^{a} \rho\left(A^{\prime} \dot{u}_{0 x}{ }^{2}+A^{\prime} \dot{u}_{y}{ }^{2}+A^{\prime} \dot{u}_{z}{ }^{2}+l_{z}^{\prime} \dot{u}_{y, x}{ }^{2}+l_{y}^{\prime} \dot{u}_{z, x}{ }^{2}+l_{\lambda}^{\prime} \dot{\theta}^{2}+I_{\omega} \dot{\theta}_{, x}{ }^{2}+I_{\theta_{y}}^{\prime} \dot{\theta}^{2}+\right. \\
\left.I_{v_{z}}^{\prime} \dot{\theta}^{2}-2 S_{y}^{\prime} \dot{u}_{0 x} \dot{u}_{z, x}+2 l_{z z}^{\prime} \dot{u}_{y, x} \dot{\theta}+2 I_{\lambda \omega}^{\prime} \dot{\theta} \dot{\theta}_{x, x}+2 S_{\hat{y}_{y}}^{\prime} \dot{u}_{y} \dot{\theta}\right) d x,
\end{gathered}
$$

where

$$
\begin{gathered}
\vartheta_{y}=-\left(z-C_{z}\right), \vartheta_{z}=\left(y-C_{y}\right) \\
\lambda=\left(y C_{z, x}-z C_{y, x}\right) \\
A^{\prime}=\int_{S} d A^{\prime}, S_{y}^{\prime}=\int_{S} z d A^{\prime}, \\
S_{\vartheta_{y}}^{\prime}=\int_{S} \vartheta_{y} d A^{\prime}, \\
I_{y}^{\prime}=\int_{S} z^{2} d A^{\prime}, I_{z}^{\prime}=\int_{S} y^{2} d A^{\prime}, \\
I_{\omega}^{\prime}=\int_{S} \omega^{2} d A^{\prime}, I_{\lambda}^{\prime}=\int_{S} \lambda^{2} d A^{\prime}, \\
I_{\vartheta_{y}}^{\prime}=\int_{S} \vartheta_{y}^{2} d A^{\prime}, I_{\vartheta_{z}}^{\prime}=\int_{S} \vartheta_{z}^{2} d A^{\prime} \\
I_{\lambda z}^{\prime}=\int_{S} y \lambda d A^{\prime}, I_{\lambda \omega}^{\prime}=\int_{S} \lambda \omega d A^{\prime} .
\end{gathered}
$$

Parameter $d A^{\prime}$ in formula (36) is defined using the formula

$$
d A^{\prime}=g \sqrt{1+\beta^{2}} d s=\sqrt{1+\beta^{2}} d A .
$$

Using Hamilton's principle (29), one gets the following system of displacement equations describing the thinwalled beam vibration problem:

$$
\begin{gathered}
E^{\prime}\left(-A^{*} u_{0 x, x}+S_{y}^{*} u_{z, x x}\right)_{, x}-\rho\left(-A^{\prime} \ddot{u}_{0 x}+S_{y}^{\prime} \ddot{u}_{z, x}\right)=0, \\
E^{\prime}\left(I_{z}^{*} u_{y, x x}+I_{\psi z}^{*} \theta_{, x}+I_{\delta z}^{*} \theta\right)_{, x x}-\rho\left(-A^{\prime} \ddot{u}_{y}-S_{v_{y}}^{\prime} \ddot{\theta}+\left(I_{z}^{\prime} \ddot{u}_{y, x}+I_{\lambda z}^{\prime} \ddot{)_{, x}}\right)=0,\right.
\end{gathered}
$$




$$
\begin{aligned}
& E^{\prime}\left(-S_{y}^{*} u_{0 x, x}+I_{y}^{*} u_{z, x x}\right)_{, x x}-\rho\left(-A^{\prime} \ddot{u}_{z}+\left(I_{y}^{\prime} \ddot{u}_{z, x}-S_{y}^{\prime} \ddot{u}_{0 x}\right)_{, x}\right)=0, \\
& E^{\prime}\left(\left(I_{\omega}^{*} \theta_{x x}+I_{\psi \omega}^{*} \theta_{, x}+I_{\delta \omega}^{*} \theta\right)_{x x}+\left(-I_{\psi}^{*} \theta_{x}-I_{\psi z}^{*} u_{y, x x}-I_{\psi \omega}^{*} \theta_{x x}-I_{\delta \psi}^{*} \theta\right)_{, x}+I_{\delta}^{*} \theta+\right. \\
& \left.I_{\delta z}^{*} u_{y, x x}+I_{\delta \omega}^{*} \theta_{x x}+I_{\delta \psi}^{*} \theta_{x}\right)-\left(G I_{s} \theta_{x}\right)_{x}-\rho\left(-I_{\lambda}^{\prime} \ddot{\theta}-I_{\theta_{y}}^{\prime} \ddot{\theta}-I_{\theta_{z}}^{\prime} \ddot{\theta}-I_{\lambda z}^{\prime} \ddot{u}_{y, x}-I_{\lambda \omega}^{\prime} \ddot{\theta}_{x}-\right. \\
& S_{v_{y}}^{\prime} \ddot{u}_{y}-S_{\vartheta_{z}}^{\prime} \ddot{u}_{z}+\left(I_{\omega}^{\prime} \ddot{\theta}_{x}+I_{\lambda \omega}^{\prime} \ddot{\theta}_{, x}\right)=0,
\end{aligned}
$$

and associated with the system of differential equations (38)-(41), equations determining the boundary conditions,

$$
\left[N \delta u_{0 x}\right]_{-a}^{a}=0,
$$

where $N=E^{\prime}\left(A^{*} u_{0 x, x}-S_{y}^{*} u_{z, x x}\right)$,

$$
\left[M_{z} \delta u_{y, x}\right]_{-a}^{a}=0,\left[M_{y} \delta u_{z, x}\right]_{-a}^{a}=0,
$$

where

$$
\begin{gathered}
M_{z}=-E^{\prime}\left(I_{z}^{*} u_{y, x x}+I_{\psi z}^{*} \theta_{, x}+I_{\delta z}^{*} \theta\right), \\
M_{y}=-E^{\prime}\left(-S_{y}^{*} u_{0 x, x}+I_{y}^{*} u_{z, x x}\right) . \\
{\left[Q_{y} \delta u_{y}\right]_{-a}^{a}=0,\left[Q_{z} \delta u_{z}\right]_{-a}^{a}=0,}
\end{gathered}
$$

where

$$
\begin{gathered}
Q_{y}=-E^{\prime}\left(I_{z}^{*} u_{y, x x}+I_{\psi z}^{*} \theta_{, x}+I_{\delta z}^{*} \theta\right)_{, x}-\rho\left(-I_{z}^{\prime} \ddot{u}_{y, x}-I_{\lambda z}^{\prime} \ddot{\theta}\right), \\
Q_{z}=-E^{\prime}\left(-S_{y}^{*} u_{0 x, x}+I_{y}^{*} u_{z, x x}\right)_{, x}-\rho\left(-I_{y}^{\prime} \ddot{u}_{z, x}+S_{y}^{\prime} \ddot{u}_{0 x}\right), \\
{\left[B \delta \theta_{, x}\right]_{-a}^{a}=0,}
\end{gathered}
$$

where

$$
\begin{gathered}
B=-E^{\prime}\left(I_{\omega}^{*} \theta_{, x x}+I_{\psi z}^{*} u_{y, x x}+I_{\psi \omega}^{*} \theta_{, x}+I_{\delta \omega}^{*} \theta\right), \\
{\left[M_{x} \delta \theta\right]_{-a}^{a}=0,}
\end{gathered}
$$

where $\quad M_{x}=B_{, x}+E^{\prime}\left(I_{\psi}^{*} \theta_{, x}+I_{\psi z}^{*} u_{y, x x}+I_{\psi \omega}^{*} \theta_{, x x}+I_{\delta \psi}^{*} \theta\right)+$ $G I_{s} \theta_{, x}-\rho\left(-I_{\omega}^{\prime} \ddot{\theta}_{, x}-I_{\lambda \omega}^{\prime} \ddot{\theta}\right)$.

\section{Problem solution}

Solutions $u_{0 x}(x), u_{y}(x), u_{z}(x), \theta(x)$ of the system of differential equations (38)-(41) will be sought as expansions of the functions into series relative to the Legendre polynomials [8]

$$
\begin{gathered}
u_{\beta}(x)=\sum_{k=0}^{\infty} a_{k}\left[u_{\beta}\right] P_{k}\left(\frac{x}{a}\right), \beta=0 x, y, z ; \\
\theta=\sum_{k=0}^{\infty} a_{k}[\theta] P_{k}\left(\frac{x}{a}\right)
\end{gathered}
$$

where $P_{k}(x)$ are the Legendre polynomials defined using the formula

$$
P_{k}(x)=2^{-k} \sum_{i=0}^{[k / 2]}(-1)^{i}\left(\begin{array}{c}
k \\
i
\end{array}\right)\left(\begin{array}{c}
2 k-2 i) \\
k
\end{array}\right) x^{k-2 i}
$$

or using the following recurrence formula:

$$
\begin{aligned}
P_{0}(x) & =1, P_{1}(x)=x, \\
P_{k+1}(x) & =\frac{2 k+1}{k+1} x P_{k}(x)-\frac{n}{n+1} P_{k-1}(x) .
\end{aligned}
$$

Legendre polynomials $P_{k}(x)$ are a special kind of Gegenbauer polynomials $C_{k}^{(\lambda)}(x)$ and dependence $P_{k}(x)=C_{k}^{\left(\frac{1}{2}\right)}(x)$ holds between them.

Let us use the following theorem, derived and presented by Lewanowicz in, amongst others, [9], to determine the coefficients of series (47). This theorem describes the method of determining the solutions of differential equations (in the form of the Gegenbauer series) with variable coefficients.

Theorem: If the function $\boldsymbol{f}$ satisfies a linear differential equation of order $n>0$.

$$
\sum_{i=0}^{n} \mathbf{P}_{i}(x) \mathbf{f}^{(i)}(x)=\mathbf{P}(x)
$$

and

$$
\mathbf{Q}_{i}(x)=\sum_{j=i}^{n}(-1)^{j-i}\left(\begin{array}{c}
j \\
j-i
\end{array}\right)\left(\mathbf{P}_{j}(x)\right)^{(j-i)}, i=0,1, \ldots, n
$$

and functions $\left(\mathbf{Q}_{0}(x) \boldsymbol{f}\right)^{(n)},\left(\mathbf{Q}_{1}(x) \boldsymbol{f}\right)^{(n-1)}, \ldots, \mathbf{Q}_{n}(x) \boldsymbol{f}, \boldsymbol{P}$ have specific Gegenbauer series coefficients, then for each integral $k$, the following identity holds true:

$\sum_{i=0}^{n} 2^{i} \sum_{m=0}^{n-i} \varrho_{n i m}(k) c_{k-n+i+2 m}\left[\mathbf{Q}_{i}(x) \mathbf{f}(x)\right]=\sum_{m=0}^{n} \varrho_{n 0 m}(k) c_{k-n+2 m}[\mathbf{P}(x)]$ fork $\geq n$

where $\varrho_{i j m}(k)$ are functions of integral variable $k$.

$$
\begin{gathered}
\varrho_{i j m}(k)=(-1)^{m}\left(\begin{array}{c}
i-j \\
m
\end{array}\right)(k+\lambda-i)_{j+m}(k+\lambda-i+j+2 m)(k+ \\
+\lambda+m+1)_{i-m}\left[(k+\lambda)^{2}-i^{2}\right]^{-1} \\
(\alpha)_{0}=1,(\alpha)_{k}=\alpha(\alpha+1) \ldots(\alpha+k-1) \text { for } k \geq 1
\end{gathered}
$$




$$
c_{k}[\boldsymbol{h}]=(k+\lambda)^{-1} a_{k}[\boldsymbol{h}],
$$

and $a_{k}[\boldsymbol{h}]$ is the $k$ th coefficient of the expansion of the function $\boldsymbol{h}(x)$ into a series relative to Gegenbauer polynomials $C_{k}^{(\lambda)}(x)(\lambda \neq 0)$ (a proof of this theorem can be found in [9]). As already mentioned, in a special case, when $\lambda=1 / 2$, the Gegenbauer polynomials become Legendre polynomials $P_{k}(x)=C_{k}^{\left(\frac{1}{2}\right)}(x)$.

Expansion coefficients $c_{k}\left[\mathbf{Q}_{i}(x) \mathbf{f}(x)\right]$ of the function product $\mathbf{Q}_{i}(x) \quad \mathbf{f}(x)$ are calculated from the formula given below ([9]), assuming that matrices $\mathbf{Q}_{i}(x)$ contain exclusively polynomials. If this assumption is not satisfied, one should beforehand apply an appropriate polynomial approximation to matrices $\mathbf{Q}_{i}(x)$. For $\lambda=1 / 2$,

$$
c_{k}\left[x^{l} f(x)\right]=2^{-l} \sum_{j=0}^{l} \alpha_{l j}(k) c_{k-l+2 j}[f(x)] \text { for } k, l \geq 0
$$

where

$$
\begin{aligned}
& \alpha_{00}(k)=1 \\
& \alpha_{l j}(k)= \begin{cases}\alpha(k) \alpha_{l-1,0}(k-1) & \text { when } j=0 \\
\alpha(k) \alpha_{l-1, j}(k-1)+\beta(k) \alpha_{l-1, j-1}(k+1) \text { when } j=1,2, \ldots, l-1 \mathrm{i} l \geq 1 \\
\beta(k) \alpha_{l-1, l-1}(k+1) & \text { when } j=l\end{cases} \\
& \alpha(k)=\frac{2 k}{(2 k+1)}, \beta(k)=2-\alpha(k) .
\end{aligned}
$$

When calculating coefficients with negative subscripts, the following relations are also used if $2 \lambda=m$ is a nonnegative integer.

$$
c_{-k}[f]=\left\{\begin{array}{l}
0 \quad \text { for } k=1,2, \ldots, m-1 \\
c_{k-m}[f] \text { for } k \geq m
\end{array} .\right.
$$

The problem of free vibrations described by equations (38)-(41) can be described by the following matrix differential equation of the fourth order:

$$
\sum_{i=0}^{4}\left(\mathbf{P}_{i}(x)-\omega^{2} \mathbf{R}_{i}(x)\right) \mathbf{f}^{(i)}(x)=\mathbf{0} .
$$

In the considered problem, matrices $\mathbf{P}_{i}$ occurring in formula (59) assume the following form:

$$
\begin{aligned}
& \mathbf{P}_{0}=E\left[\begin{array}{cccc}
0 & 0 & 0 & 0 \\
0 & 0 & 0 & k_{24}^{0} \\
0 & 0 & 0 & 0 \\
0 & 0 & 0 & k_{44}^{0}
\end{array}\right] \mathbf{P}_{1}=E\left[\begin{array}{cccc}
k_{11}^{1} & 0 & 0 & 0 \\
0 & 0 & 0 & k_{24}^{1} \\
k_{31}^{1} & 0 & 0 & 0 \\
0 & 0 & 0 & k_{44}^{1}
\end{array}\right] \mathbf{P}_{2}=E\left[\begin{array}{cccc}
k_{11}^{2} & 0 & k_{13}^{2} & 0 \\
0 & k_{22}^{2} & 0 & k_{24}^{2} \\
k_{31}^{2} & 0 & k_{33}^{2} & 0 \\
0 & k_{42}^{2} & 0 & k_{44}^{2}
\end{array}\right] \\
& \mathbf{P}_{3}=E\left[\begin{array}{cccc}
0 & 0 & k_{13}^{3} & 0 \\
0 & k_{22}^{3} & 0 & k_{24}^{3} \\
k_{31}^{3} & 0 & k_{33}^{3} & 0 \\
0 & k_{42}^{3} & 0 & k_{44}^{3}
\end{array}\right] \mathbf{P}_{4}=E\left[\begin{array}{cccc}
0 & 0 & 0 & 0 \\
0 & k_{22}^{4} & 0 & 0 \\
0 & 0 & k_{33}^{4} & 0 \\
0 & 0 & 0 & k_{44}^{4}
\end{array}\right] \\
& \mathbf{R}_{0}=\rho\left[\begin{array}{cccc}
m_{11}^{0} & 0 & 0 & 0 \\
0 & m_{22}^{0} & 0 & m_{24}^{0} \\
m_{31}^{0} & 0 & m_{33}^{0} & 0 \\
0 & m_{42}^{0} & 0 & m_{44}^{0}
\end{array}\right] \mathbf{R}_{1}=\rho\left[\begin{array}{cccc}
0 & 0 & m_{13}^{1} & 0 \\
0 & m_{22}^{1} & 0 & m_{24}^{1} \\
m_{31}^{1} & 0 & m_{33}^{1} & 0 \\
0 & m_{42}^{1} & 0 & m_{44}^{1}
\end{array}\right] \mathbf{R}_{2}=\rho\left[\begin{array}{cccc}
0 & 0 & 0 & 0 \\
0 & m_{22}^{2} & 0 & 0 \\
0 & 0 & m_{33}^{2} & 0 \\
0 & 0 & 0 & m_{44}^{2}
\end{array}\right] \\
& \mathbf{R}_{3}=\rho\left[\begin{array}{llll}
0 & 0 & 0 & 0 \\
0 & 0 & 0 & 0 \\
0 & 0 & 0 & 0 \\
0 & 0 & 0 & 0
\end{array}\right] \mathbf{R}_{4}=\rho\left[\begin{array}{llll}
0 & 0 & 0 & 0 \\
0 & 0 & 0 & 0 \\
0 & 0 & 0 & 0 \\
0 & 0 & 0 & 0
\end{array}\right]
\end{aligned}
$$

where the elements of the matrices are defined using formulas

$$
\begin{gathered}
k_{24}^{0}=I_{\delta z, x x}^{*} \\
k_{44}^{0}=I_{\delta}^{*}-I_{\delta \psi, x}^{*}+I_{\delta \omega, x x}^{*} \\
k_{11}^{1}=-A_{, x}^{*} \\
k_{24}^{1}=2 I_{\delta z, x}^{*}+I_{\psi z, x x}^{*} \\
k_{31}^{1}=-S_{y, x x}^{*} \\
k_{44}^{1}=2 I_{\delta \omega, x}^{*}-I_{\psi, x}^{*}+I_{\psi \omega, x x}^{*}-\frac{G}{E} I_{S, x} \\
k_{11}^{2}=-A^{*} \\
k_{13}^{2}=-S_{y, x}^{*} \\
k_{22}^{2}=I_{z, x x}^{*} \\
k_{24}^{2}=I_{\delta z}^{*}+2 I_{\psi z, x}^{*}+I_{\omega z, x x}^{*} \\
k_{31}^{2}=-2 S_{y, x}^{*} \\
k_{33}^{2}=I_{y, x x}^{*} \\
k_{42}^{2}=I_{\delta z}^{*}-I_{\psi z, x}^{*}+I_{\omega z, x x}^{*}
\end{gathered}
$$

$$
\begin{gathered}
m_{13}^{1}=-S_{y}^{\prime} \\
m_{22}^{1}=-I_{z, x}^{\prime} \\
m_{24}^{1}=-I_{\lambda z}^{\prime} \\
m_{31}^{1}=S_{y}^{\prime} \\
m_{33}^{1}=-I_{y, x}^{\prime} \\
m_{42}^{1}=I_{\lambda z}^{\prime} \\
m_{44}^{1}=I_{\omega, x}^{\prime}
\end{gathered}
$$




$$
\begin{aligned}
& m_{22}^{2}=-I_{z}^{\prime} \\
& m_{33}^{2}=-I_{y}^{\prime} \\
& m_{44}^{2}=-I_{\omega}^{\prime}
\end{aligned}
$$

In the case of the fourth order $(n=4)$ differential equation, infinite system of equations (52) describing the interdependences between the coefficients of the expansion of soughtfunction $(\mathrm{x})=\left[u_{0 x}(x), u_{y}(x), u_{z}(x), \theta(x)\right]^{T}$, is defined using the formula

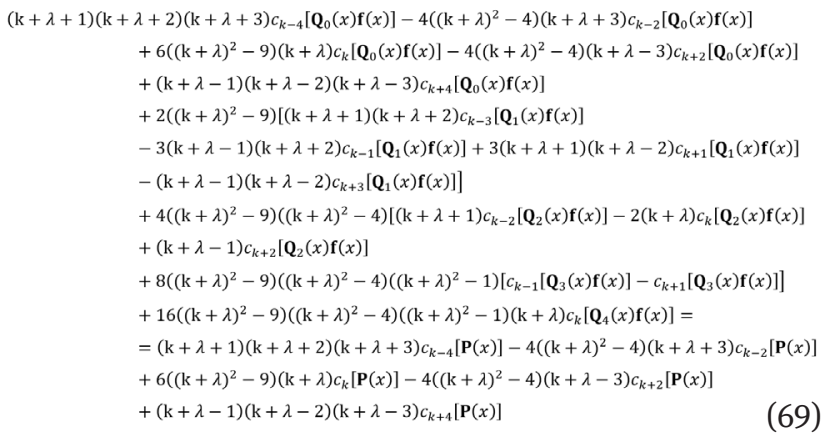

where $\lambda=1 / 2$.

The system, satisfied at $k \geq 4$, should be completed with 14 equations describing boundary conditions. In order to formulate the conditions, the following relations defining the values of the Legendre' polynomials and their derivatives in points $x= \pm 1$ are used:

$$
\begin{gathered}
P_{k}(1)=1, P_{k}(-1)=(-1)^{k} \\
d^{m} P_{k}(x) /\left.d x^{m}\right|_{x=1}=(2 m-1) ! !\left(\begin{array}{l}
k+m \\
k-m
\end{array}\right) \\
d^{m} P_{k}(x) /\left.d x^{m}\right|_{x=-1}=(-1)^{k-m} d^{m} P_{k}(x) /\left.d x^{m}\right|_{x=1}
\end{gathered}
$$

Exemplary equations describing boundary condition $\left.u_{z}(x)\right|_{x=a}=0$ have the form

$\left.u_{z}(x)\right|_{x=a}=\sum_{k=0}^{\infty} a_{k}\left[u_{z}\right] P_{k}(a / a)=\sum_{k=0}^{\infty}(-1)^{k}(k+1 / 2) c_{k}\left[u_{z}\right]=0$.

\section{Calculation example}

In order to illustrate the proposed method as well as to verify it and show its effectiveness, a numerical example is provided. In the example, the eigenproblem was solved using Legendre polynomials for approximation. The sought functions were approximated with 15 series terms. The Wolfram Mathematica 10 [23] software was used for the calculations.

\subsection{Example}

Nonprismatic monosymmetric I-beams with a linearly variable web height are analysed. Two static systems, that is, a clamped-clamped beam and a cantilever beam, are considered. The diagrams of the beams and the dimensions of their cross sections are shown in Fig. 4. The material parameters of the beams are $E=210 \mathrm{GPa}, G=80.77 \mathrm{GPa}$ and $\rho=7850 \mathrm{~kg} / \mathrm{m}^{3}$.

Formula (28) derived in this article was used to calculate the geometrical characteristics of the cross section. Various size of approximation base was used for checking the convergence of the presented method. The sought functions (47) were approximated with 10, 15 and 20 series terms. The obtained results are presented in Tables 1 and 2.

In order to verify the model and the effectiveness of the presented method of solving the derived equations, the considered problem was solved using FEM. The Abaqus program, in which $0.02 \mathrm{~m} \times 0.02 \mathrm{~m}$ rectangular shell elements were adopted for the calculations, and the SOFiSTiK program, in which the beams were solved using thin-walled beam elements with 7 degrees of freedom (DOFs), were used for the calculations. In the SOFiSTiK calculations, various numbers of elements were used. The beams were divided into 20, 25 and 50 elements. The obtained results for the above two cases and the results yielded by FEM are presented in Tables 1 and 2 .

In the case of the solutions obtained using the shell elements (Abaqus), 'additional' local effects, consisting in local deformations of the medium, appear in the eigenforms corresponding to higher frequencies. In the tables, the forms are denoted as lf - 'local form'.

To examine the impact of considering the so-called reduced thickness $g^{*}=\left(1+\beta^{2}\right)^{-\frac{3}{2}} g$ in definition of the geometric property of an area, the calculations were done in two ways: (1) the characteristics were calculated from formulas (28), that is, using reduced thickness $g^{\star}$, and (2) assuming that $\beta=0$, that is, $g^{\star}=g$. By analysing the two cases, it was possible to evaluate the influence of the simplified method of determining the cross-sectional characteristics on the eigenfrequency values. Moreover, the influence of the generalisation introduced into the model (versus the Wilde model) to take into account the variability in the location of the shear centres in the cross sections, described by functions $C_{z}(x), C_{y}(x)$, was examined. For this purpose, calculations for the Wilde model were also performed (see [22]). omitting the components in which derivatives $d^{k} C_{z}(x) / d x^{k}, d^{k} C_{k}(x) / d x^{k}$ appear as factors in all the derived formulas. In the calaculations, $d^{k} C_{z}(x) / d x^{k} \equiv 0$ (in the case of monosymmetric cross 

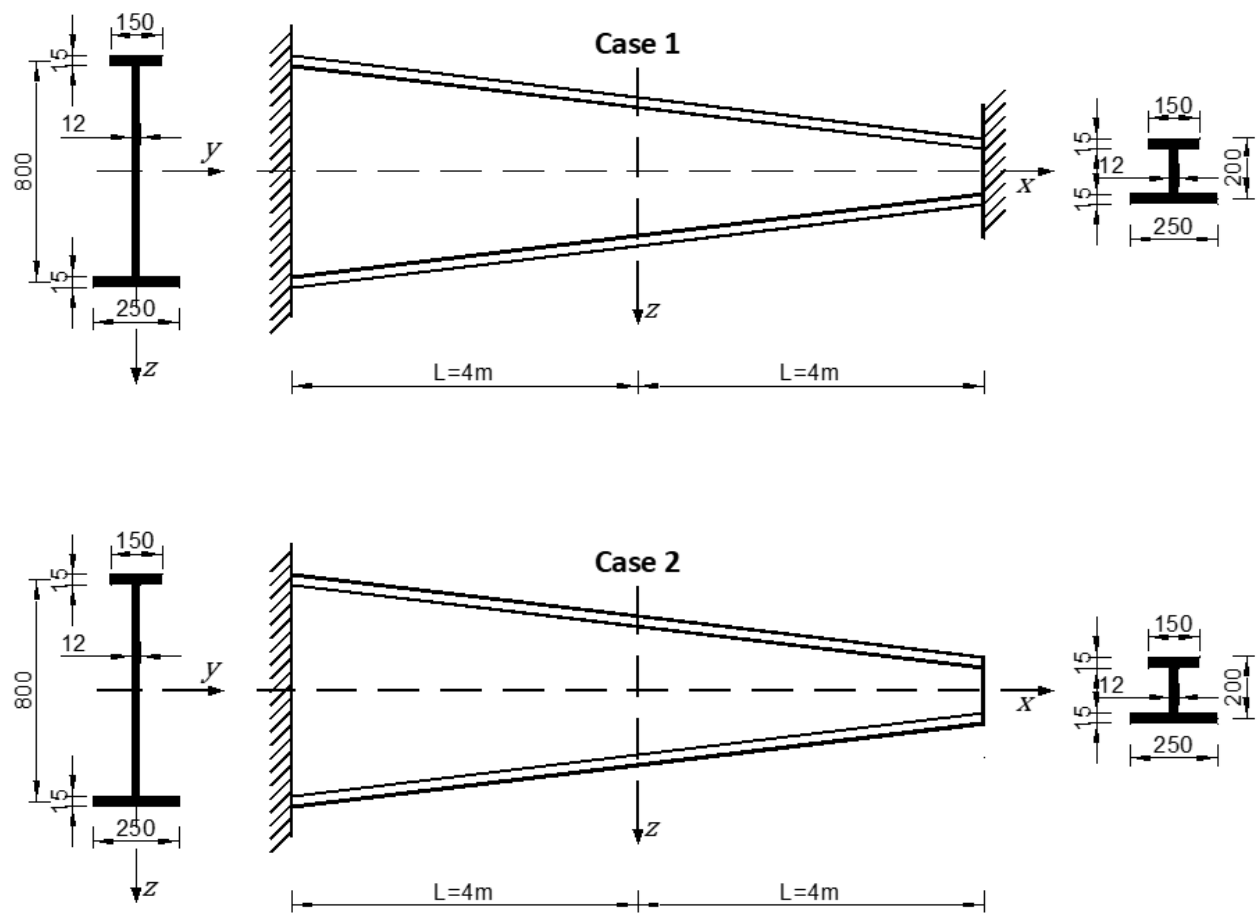

Figure 4: Analysed beams and dimensions of their cross sections.

Table 1: Eigenfrequencies in case of clamped-clamped support (C-C) [Hz].

\begin{tabular}{|c|c|c|c|c|c|c|c|}
\hline Mode number & $\begin{array}{l}\text { Paper } \\
\operatorname{dim}=10\end{array}$ & $\begin{array}{l}\text { Paper } \\
\operatorname{dim}=15\end{array}$ & $\begin{array}{l}\text { Paper } \\
\operatorname{dim}=20\end{array}$ & $\begin{array}{l}\text { FEM SOFiSTiK } \\
\text { beam element 7DOF } \\
\mathrm{d}=\mathbf{2 0}\end{array}$ & $\begin{array}{l}\text { FEM SOFiSTiK } \\
\text { beam element 7DOF } \\
d=25\end{array}$ & $\begin{array}{l}\text { FEM SOFiSTiK } \\
\text { beam element 7DOF } \\
d=50\end{array}$ & $\begin{array}{l}\text { FEM Abaqus } \\
\text { shell elements } \\
\text { (quad) }\end{array}$ \\
\hline 1 & 10.17 & 10.15 & 10.15 & 10.17 & 10.16 & 10.15 & 10.05 \\
\hline 2 & 18.43 & 18.39 & 18.39 & 17.93 & 17.92 & 17.89 & 18.18 \\
\hline 3 & 26.70 & 26.68 & 26.68 & 26.83 & 26.75 & 26.64 & 26.11 \\
\hline 4 & 48.69 & 48.70 & 48.69 & 47.62 & 47.45 & 47.20 & 46.79 (If) \\
\hline 5 & 52.71 & 50.72 & 50.72 & 49.49 & 49.41 & 49.31 & 48.18 \\
\hline 6 & 53.11 & 52.92 & 52.92 & 51.86 & 51.57 & 51.17 & 49.32 \\
\hline 7 & 90.71 & 82.42 & 82.34 & 83.58 & 82.66 & 81.43 & 67.38 (If) \\
\hline 8 & 96.80 & 93.59 & 93.59 & 94.05 & 93.45 & 92.64 & 85.73 (If) \\
\hline
\end{tabular}

sections, $\left.C_{\mathrm{y}}(\mathrm{x}) \equiv 0\right)$ was assumed. The approximation with 15 series terms was used in comparative calculations. The obtained results are presented in Tables 3 and 4.

\section{Conclusions}

The following conclusions can be drawn from the results presented in the examples:

- using the proposed method, one can solve problems relating to the dynamics of nonprismatic thin-walled beams with any geometry parameters. Numerical examples show that the method is highly accurate and efficient. Only approximation with 15 series terms for each calculated function has to be used to obtain sufficient results (60 series terms for all functions). In case of FEM, the total number of finite elements required for analysis is circa 25 . It equals 175 degrees of freedom. Moreover, the presented semi-analytical method is useful for further calculation which is difficult in case of FEM. 
Table 2: Eigenfrequencies in case of clamped-free support (C-F) [Hz].

\begin{tabular}{|c|c|c|c|c|c|c|c|}
\hline Mode number & $\begin{array}{l}\text { Paper } \\
\operatorname{dim}=10\end{array}$ & $\begin{array}{l}\text { Paper } \\
\operatorname{dim}=15\end{array}$ & $\begin{array}{l}\text { Paper } \\
\operatorname{dim}=\mathbf{2 0}\end{array}$ & $\begin{array}{l}\text { FEM SOFiSTiK } \\
\text { beam element 7DOF } \\
d=20\end{array}$ & $\begin{array}{l}\text { FEM SOFiSTiK } \\
\text { beam element 7DOF } \\
d=25\end{array}$ & $\begin{array}{l}\text { FEM SOFISTiK } \\
\text { beam element 7DOF } \\
d=50\end{array}$ & $\begin{array}{l}\text { FEM Abaqus } \\
\text { shell elements } \\
\text { (quad) }\end{array}$ \\
\hline 1 & 2.20 & 2.19 & 2.20 & 2.15 & 2.15 & 2.15 & 2.19 \\
\hline 2 & 8.33 & 7.68 & 7.60 & 7.68 & 7.69 & 7.69 & 7.55 \\
\hline 3 & 13.44 & 12.96 & 12.91 & 12.26 & 12.25 & 12.24 & 12.77 \\
\hline 4 & 14.42 & 13.88 & 13.85 & 13.66 & 13.67 & 13.68 & 13.77 \\
\hline 5 & 24.31 & 23.18 & 23.13 & 23.97 & 23.95 & 23.93 & 22.75 \\
\hline 6 & 35.95 & 32.68 & 32.54 & 31.07 & 31.00 & 30.90 & 30.89 \\
\hline 7 & 44.18 & 50.31 & 50.21 & 52.13 & 51.91 & 51.60 & 48.68 lf) \\
\hline 8 & 61.28 & 59.55 & 59.30 & 57.68 & 57.46 & 57.17 & 52.96 (If) \\
\hline
\end{tabular}

Table 3: Eigenfrequencies for different models in case of clampedclamped support (C-C) [Hz].

\begin{tabular}{llll}
\hline Mode number & Paper & $\begin{array}{l}\text { Paper } \\
\boldsymbol{\beta}=\mathbf{0}\end{array}$ & $\begin{array}{l}\text { Paper } \\
\boldsymbol{C}_{\mathbf{z}}{ }^{\circ}=\mathbf{0}\end{array}$ \\
\hline 1 & 10.15 & 10.16 & 9.99 \\
2 & 18.39 & 18.41 & 18.63 \\
3 & 26.68 & 26.71 & 26.55 \\
4 & 48.70 & 48.76 & 48.21 \\
5 & 50.72 & 50.78 & 51.45 \\
6 & 52.92 & 52.97 & 52.92 \\
7 & 82.42 & 82.52 & 81.84 \\
8 & 93.59 & 93.70 & 94.47 \\
\hline
\end{tabular}

Table 4: Eigenfrequencies for different models in case of clampedfree support (C-F) [Hz].

\begin{tabular}{llll}
\hline Mode number & Paper & $\begin{array}{l}\text { Paper } \\
\boldsymbol{\beta}=\mathbf{0}\end{array}$ & $\begin{array}{l}\text { Paper } \\
\boldsymbol{C}_{\mathbf{5}}{ }^{\circ}=\mathbf{0}\end{array}$ \\
\hline 1 & 2.19 & 2.20 & 2.21 \\
2 & 7.68 & 7.68 & 7.60 \\
3 & 12.96 & 12.97 & 13.36 \\
4 & 13.88 & 13.89 & 13.88 \\
5 & 23.18 & 23.21 & 22.44 \\
6 & 32.68 & 32.71 & 33.91 \\
7 & 50.31 & 50.37 & 48.72 \\
8 & 59.55 & 59.61 & 61.33 \\
\hline
\end{tabular}

- in the considered free vibration problem, the adoption of the simplified way of determining the geometrical characteristics of the cross section has no significant effect on the values of the eigenfrequencies of the considered systems;

- the differences between the eigenfrequency values determined using, respectively, the Wilde model and the generalised model are not significantly large (for the first eight eigenfrequencies, they amount to $0-1.6 \%$ for the clamped-clamped beam and to $0-3.8 \%$ for the clamped-free beam). A more detailed analysis shows that the omission of the derivatives of functions $C_{\mathrm{z}}(x), C_{\mathrm{y}}(x)$ generates the largest differences in the case of the frequencies corresponding to the forms in which torsion dominates. One can expect that for beams with any cross section (no monosymmetry), the differences will probably be larger.

\section{References}

[1] Ambrosini, R. D., Riera, J. D. \& Danesi, R. F. (2000). A modified Vlasov theory for dynamic analysis of thin-walled and variable open section beams. Engineering Structures. 22(8), 890-900. DOI: 0.1016/S0141-0296(99)00043-7.

[2] Arpaci, A. \& Bozdag, S. E. (2002). On free vibration analysis of thin-walled beams with nonsymmetrical open cross-sections. Computers and Structures. 80(7-8), 691-695.

[3] Asgarian, B., Soltani, M. \& Mohri, F. (2013). Lateral-torsional buckling of tapered thin-walled beams with arbitrary crosssections. Thin-Walled Structures. 62,96-108. DOI: 10.1016/j. tws.2012.06.007.

[4] Borbón, F. \& Ambrosini, D. (2010). On free vibration analysis of thin-walled beams axially loaded. Thin-Walled Structures. 48(12), 915-920. DOI: 10.1016/j.tws.2010.06.002.

[5] Chen, C. N. (1998). Variational derivation of the dynamic equilibrium equations of nonprismatic thin-walled beams defined on an arbitrary coordinate system. Mechanics of Structures and Machines. 26(2),219-237. 
[6] Eisenberger. M. (1997). Torsional vibrations of open and variable cross-section bars. Thin-Walled Structures.28(3-4), 269-278.

[7] Heyliger, P. R. (2015). Elasticity-based free vibration of anisotropic thin-walled beams. Thin-Walled Structures.95:73-87. DOI: 10.1016/j.tws.2015.06.014.

[8] Lebiediew, N. N. (1957). Special Functions and their applications. Warsaw: PWN; (in Polish).

[9] Lewanowicz, S. (1976). Construction of a recurrence relation of the lowest order for coefficients of the Gegenbauer series. Applicationes Mathematicae XV(3), 345-96.

[10] Nguyen, C. T., Moon. J., Le. V. N. \& Lee, H. (2011). Natural frequency for torsional vibration of simply supported steel I-girders with intermediate bracings. Thin-Walled Structures. 49(4), 534-542. DOI: 10.1016/j.tws.2010.12.001.

[11] Machado, S. P. \& Cortínez, V. H. (2005). Non-linear model for stability of thin-walled composite beams with shear deformation. Thin-Walled Structures. 43(10),1615-1645. DOI: 10.1016/j.tws.2005.06.008.

[12] Mohri, F., Brouki, A. \& Roth, J. C. (2003). Theoretical and numerical stability analyses of unrestrained, mono-symmetric thin-walled beams. Journal of Constructional Steel Research. 59(1), 63-90. DOI: 10.1016/S0143-974X(02)00007-X.

[13] Mohri, F., Damil, N. \& Ferry, M. P. (2008). Large torsion finite element model for thin-walled beams. Computers and Structures. 86(7-8), 671-683. DOI: 10.1016/j. compstruc.2007.07.007.

[14] Paszkowski, S. (1975). Numerical applications of Chebyshev polynomials. Warsaw: PWN; (in Polish).

[15] Ruta, P. \& Szybiński, J. (2014). Analysis of the free vibration of a thin-walled nonprismatic beam. Journal of Civil Engineering, Environment and Architecture. XXXI(61), 173-184 (in Polish). DOI:10.7862/rb.2014.39.

[16] Ruta, P. (1999). Application of Chebyshev series to solution of non-prismatic beam vibration problem. Journal of Sound and Vibration. 227(2), 449-467. DOI: 10.1006/jsvi.1999.2348.

[17] Ruta P. (2002). Dynamic stability problem of a non-prismatic rod. Journal of Sound and Vibration. 250(3), 445-464. DOI: 10.1006/jsvi.2001.3954.

[18] Ruta, P. \&Szybiński, J. (2015). Nonlinear analysis of nonprismatic Timoshenko beam for different geometric nonlinearity models. International Journal of Mechanical Sciences. 101-102, 349-362. DOI: 10.1016/j. ijmecsci.2015.07.020.

[19] Ruta P. (2006). The application of Chebyshev polynomials to the solution of the nonprismatic Timoshenko beam vibration problems. Journal of Sound and Vibration. 296(1-2), 243-263. DOI: 10.1016/j.jsv.2006.02.011.

[20] Soltani, M., Asgarian, B. \& Mohri, F. (2014a). Elastic instability and free vibration analyses of tapered thin-walled beams by the power series method. Journal of Constructional Steel Research. 96,106-126. DOI: 10.1016/j.jcsr.2013.11.001.

[21] Soltani, M., Asgarian, B. \& Mohri, F. (2014b). Finite element method for stability and free vibration analyses of nonprismatic thin-walled beams. Thin-Walled Structures. 82, 245 261. DOI: 10.1016/j.tws.2014.04.012.

[22] Wilde, P. (1968). The torsion of thin-walled bars with variable cross-section. Archives of Mechanics. 4(20), 431-443.

[23] Wolfram Mathematica 10. Wolfram Research @Copyright 19882015. 